\title{
Cycling of dust grains through the galactic halo
}

\author{
A. Ferrara ${ }^{\dagger}$, J. Franco* ${ }^{*}$ B. Barsella ${ }^{\dagger}$, F.Ferrini ${ }^{\dagger}$ \\ + Dipartimento di Astronomia, Università di Firenze, Firenze, Italy \\ * Instituto de Astronomía, UNAM, México City, México \\ $\dagger$ Istituto di Astronomia, Università di Pisa, Pisa, Italy
}

\begin{abstract}
The analysis of the vertical motion of dust grains inside the disk of spiral gálaxies shows that grains at high latitudes $(z / H>0.3-0.5$, where $H$ is the scale height of the gaseous disk) can be removed from the gaseous disk by radiation pressure from field stars. Once the dust particles are located at the base of the galactic corona, and neglecting the presence of magnetic fields, their motion is determined by the global light and mass distributions. We follow the dynamical evolution of grains embedded in a hot galactic corona ( $T \geq 10^{5} \mathrm{~K}$ and $n \leq 10^{-2} \mathrm{~cm}^{-3}$ ), including drag and sputtering. We find that typical sputtering time scales are larger than $10^{8} \mathrm{yrs}$, and, in these times scales, the grains can travel large radial and vertical distances from their original positions. This process can remove grains from the inner part of a galaxy and send them to large galactocentric distances. The evolution resembles a dusty galactic fountain which may pollute the outer galaxy with grains and heavy elements. Similarly, for some cases the dust particles can be detached from the galactic gravitational field and they are expelled to the intergalactic medium. In these cases, the chemical pollution is not restricted to the host galaxy and may reach material which is otherwise primordial.
\end{abstract}

\section{Introduction}

Dust grains can be pushed by the radiation pressure produced by the stellar photon field and they, in turn, can transmit their momentum to the surrounding gas. Franco (1989) has demonstrated that small dusty clouds can be expelled from the disk by radiation pressure. The process, which is called "photolevitation" and requires an efficient dust-gas coupling, depends upon the cloud column density, cloud-cloud velocity dispersions, and on the vertical distribution of disk stars. In the absence of magnetic fields, the minimum gas column density for dust-gas coupling is about $N \sim 10^{19} \mathrm{~cm}^{-2}$ and dust grains can drift inside the cloud within these column densities. The clouds get flattened during the upward acceleration process and some grains may detach from the cloud. Similarly, given the patchiness of the interstellar medium, some naked grains can escape from the disk through low density "tunnels".

Here we present, as discussed in Barsella et al. (1989b), the dynamical evolution of naked grains that are able to drift into the base of the galactic corona. The properties of the interstellar drag change drastically in the transition region between the disk and the halo: the interactions with localized dense gaseous structures disappear and sputtering with the hot, but substantially less dense, coronal gas becomes more prominent.

\section{The model for the evolution of dust grains in the halo}

The equation of motion is described in detail in Barsella et al. (1989b) and contains three terms: radiation pressure, gravity and gas drag. The optical grain properties are 
taken from Draine and Lee (1984) and the gravitational field and light distributions are simulated as in Barsella et al. (1989a). The effects of the halo gas, drag and sputtering, are followed with the scheme described by Draine and Salpeter (1979). Evaporation effects due to the hard radiation field expected in the halo are not considered.

For simplicity, to maintain the variation of the physical processes to a minimum, the pressure and density of the coronal gas are assumed constant. The considered values are in the range $10^{-3}-10^{-2} \mathrm{~cm}^{-3}$ and $3 \leq \log (p / k) \leq 4$, where $p$ is the pressure and $k$ is the Boltzmann constant. These are commonly considered as typical values (see e.g. Pettini et al. , 1982; de Boer, 1985) and determine the thermodynamical properties of the coronal gas in our model. Note that the assumed values may represent upper limits for the halo, and the effects are rather severe for what concerns to sputtering and gas drag, allowing a good confidence in the results.

\section{Results}

The motion of grains has been studied for the galaxy NGC 3198. The parameters have been taken by Kent (1987). We used bare grains of astronomical silicate with an initial radius of $100 \mathrm{~nm}$, typical in a galactic environment. The initial position has been chosen at a height of $100 \mathrm{pc}$ above midplane and at a variety of galactocentric distances (from 0.1 to $2.0 \mathrm{kpc}$ ). The grain has zero initial velocity relative to the gas, and the coronal gas is assumed to rotate at the same rate of the galaxy.

The figures show the model results for NGC 3198. Fig. 1 displays the height evolution as a function of time for two different choices of pressure and density of the coronal gas: solid curves correspond to $\log (p / k)=3$ and $n=10^{-3} \mathrm{~cm}^{-3}$, and the dashed ones correspond to the same pressure but $n=10^{-2} \mathrm{~cm}^{-3}$. Each case is shown for two different initial positions, the upper and lower curves correspond to initial galactocentric distances of $0.1,1 \mathrm{kpc}$, respectively. The height $Z$ is measured in units of the halo radius, which for this galaxy is $R_{H}=33 \mathrm{kpc}$.

The evolution of the grains sizes, in nm, is shown in Fig. 2; the upper dashed curve corresponds to the initial location near the galactic center, and the lower curve to an initial galactocentric distance of $1 \mathrm{kpc}$. For the lower density case, $n=10^{-3} \mathrm{~cm}^{-3}$, there is almost no sputtering of the grains. This plot shows clearly that dust can survive for a rather long time. We have also explored models by doubling the efficiency of sputtering given by Draine and Salpeter (1979) (i.e. to mimick the eventual effect of $\mathrm{X}==$ rays and cosmic rays in the hot galactic corona). While there is no appreciable change in the evolution for grains in the lower density case,for $n=10^{-2} \mathrm{~cm}^{-3}$ the grains reach a complete desruption after $4.5 \times 10^{8} \mathrm{yrs}$.

Finally, Fig. 3 shows the radial spread of the grains starting from height $z=100 \mathrm{pc}$ and at galactocentric distances $r=0.1$ and $1 \mathrm{kpc}$. It appears that the radial position attained is of the order of the radius of the outer arm zone. It is clear from this plot that dust grains are removed from the internal regions of the galaxy, where the density of stars producing grains is higher. 


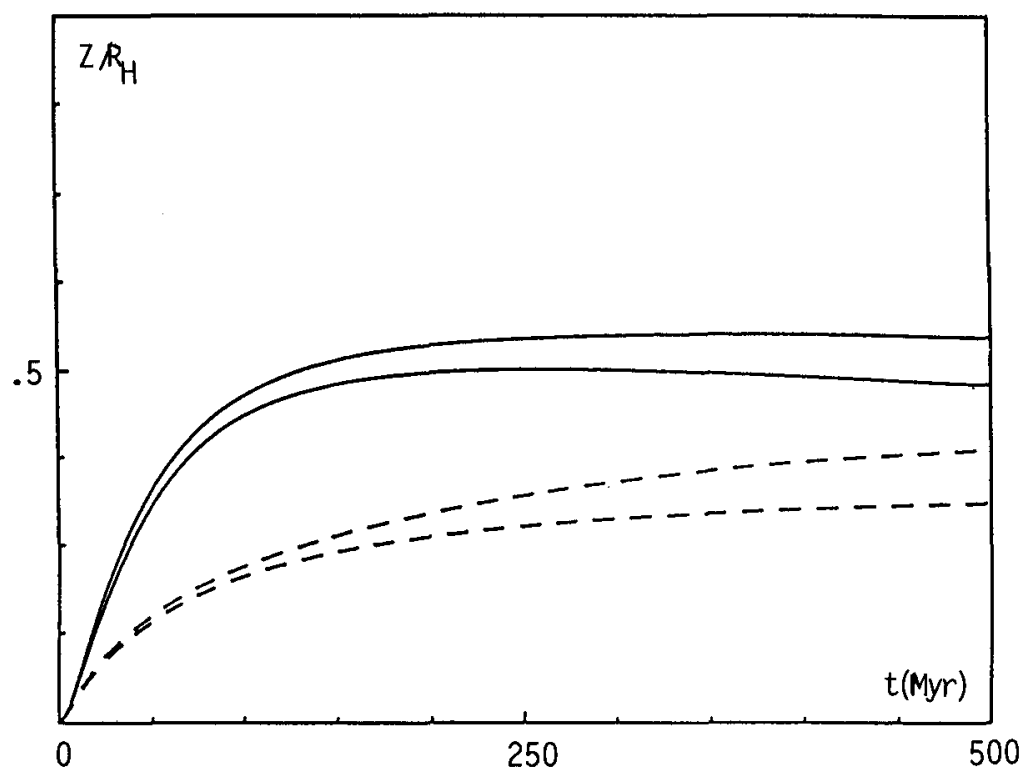

Fig. 1. Evolution of the grain height (in terms of the halo radius, $R_{H}=33 \mathrm{kpc}$ ). The solid lines correspond to $\log (p / k)=3$ and $n=10^{-3} \mathrm{~cm}^{-3}$; dashed lines correspond to the same pressure but $n=10^{-2} \mathrm{~cm}^{-3}$. The calculation has been done for a galactocentric start distance of 0.1 and $1 \mathrm{kpc}$.

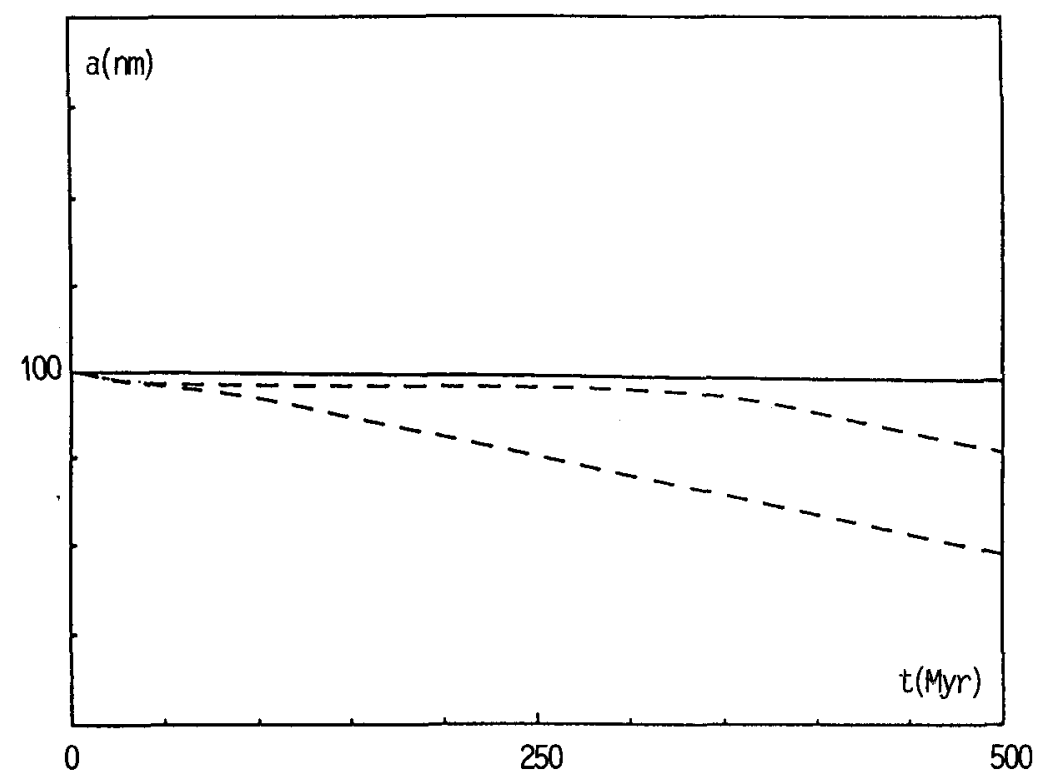

Fig. 2. Evolution of the grain radius as a function of time. The solid and dashed lines have the same meaning as in Fig. 1 


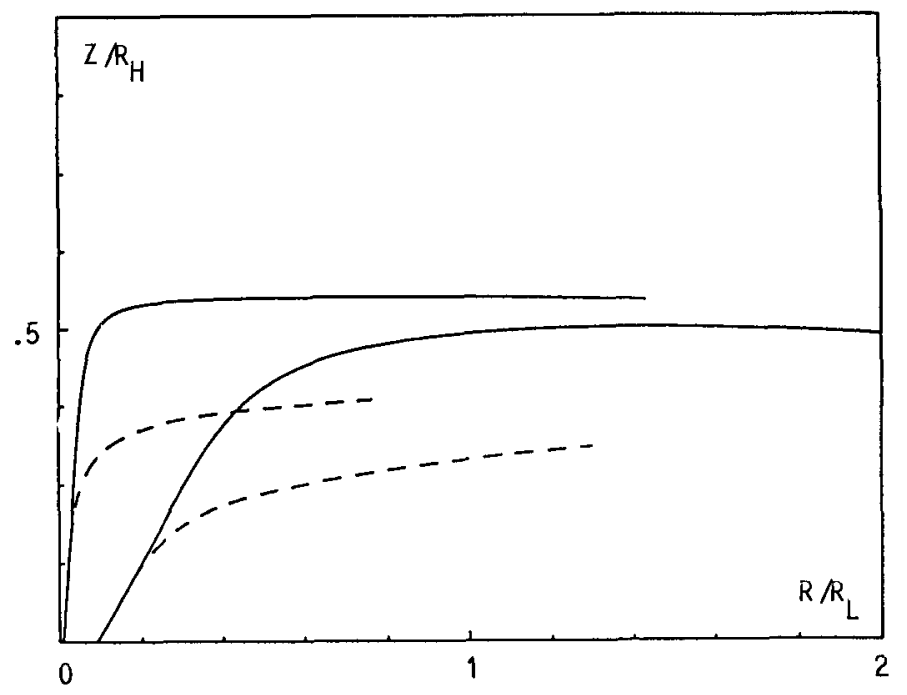

Fig. 3. $z-r$ trajectory of the grain; the ordinate is measured in units of the halo radius, the ascissa in units of the luminosity radius $R_{L}=10.8 \mathrm{kpc}$. The solid and dashed lines have the same meaning as in Fig. 1

An interesting consequence of the large.scale motion and of the erosion suffered by dust grains during their flight in the galactic halo, is the pollution of heavy elements as well as of small grains in regions that are otherwise poor in these interstellar components. The future work will concerns an extention to other galaxies, following as a guideline the static analysis of the forces by Ferrara et al. (1989), which revealed a continuity of behaviours in the galaxies, ranging from a almost complete capture of grains in the disk to their efficient expulsion out the gravitational field of the whole galaxy.

\section{References}

Barsella, B., Ferrini, F., Greenberg, J.M., and Aiello, S. 1989a, Astron. Astrophys., 209, 349

Barsella, B., Franco, J., Ferrini, F., and Ferrara, A. 1989b, in preparation

de Boer, K. S. 1985, in "The Milky Way Galaxy", IAU Symp. 106, 415, H. van Woerden et al. (eds)

Draine, B.T., and Lee, H:M. 1984, Ap. J., 285, 89

Draine, B.T., and Salpeter, E.E. 1979, Ap. J., 231, 77

Ferrara, F., Ferrini, F., Barsella, B., and Aiello, S. 1989, submitted to Astron. Astrophys.

Franco, J. 1989, preprint

Kent, S.M. 1987, Astron. J., 93, 816

Pettini, M., West, K., 1982, Ap. J., 260, 561

\section{Discussion:}

VöLK: Did you consider grain charging in the context of grain-gas coupling?.

FERRARA: No, the project is in the early phases and we are analysing the simplest cases (i.e. no B-fields, constant halo pressure and density, etc.). We will address this issue, and others, in the near future. 